Pacific Journal of Mathematic 


\title{
A CLASS OF SURJECTIVE CONVOLUTION OPERATORS
}

\author{
WOJCIECH ABRAMCZUK
}

\begin{abstract}
Let $\mu$ be a distribution with compact support in $\mathbf{R}^{n}$. In the terminology of Ehrenpreis [2] $\mu$ is called invertible for a space of distributions $\mathscr{F}$ in $\mathbf{R}^{n}$ if $\mu * \mathscr{F}=\mathscr{F}$. Using his characterisation of invertible distributions in terms of the growth of their Fourier transforms, we obtain a class of invertible distributions which properly contains the distributions with finite supports. We consider $\mathscr{F}=\mathcal{E}$ (or $\mathscr{D}^{\prime}$ ) and $\mathscr{F}=\mathscr{Q}_{F}^{\prime}$, but our results for the latter space are only partial.
\end{abstract}

1. Introduction. We follow the notation of Schwartz [6]: by $Q^{\prime}$ $\left(\mathscr{D}_{F}^{\prime}\right)$ we denote the space of distributions (distributions of finite order) in $\mathbf{R}^{n}$. $\mathcal{E}$ will denote the space of infinitely differentiable functions in $\mathbf{R}^{n}$ with the topology of uniform convergence of functions and all their derivatives on compact subsets of $\mathbf{R}^{n}$. The dual space of $\mathcal{E}$, denoted by $\mathcal{E}^{\prime}$, consists of distributions with compact support in $\mathbf{R}^{n}$. For $\mu \in \mathcal{E}^{\prime}$ we define the Fourier-Laplace transform of $\mu$ by

$$
\hat{\mu}(\zeta)=\mu\left(e^{-i\langle\cdot, \zeta\rangle}\right), \quad \zeta \in \mathbf{C}^{n} .
$$

Ehrenpreis [2] and Hörmander [3] have studied the range of convolution operators

$$
u \mapsto \mu * u, \quad \mu \in \mathcal{E}^{\prime},
$$

in each of the spaces $\mathscr{D}^{\prime}, \mathscr{D}_{F}^{\prime}$ and $\mathcal{E}$. We recall their main result: the operator (1) in $\mathcal{E}$ and, equivalently, in $\mathscr{D}^{\prime}$ (resp. in $\mathscr{Q}_{F}^{\prime}$ ) is surjective if and only if $\hat{\mu}$ is slowly decreasing (resp. very slowly decreasing) in the sense of

Definition 1 . Let $\mu \in \mathcal{E}^{\prime}$. $\hat{\mu}$ is called slowly decreasing if there exist constants $A, B$ and $m$ such that

$$
\sup _{\left|\xi-\xi_{0}\right| \leq A \log \left(2+\left|\xi_{0}\right|\right)}|\hat{\mu}(\xi)| \geq B\left(1+\left|\xi_{0}\right|\right)^{-m}
$$

for all $\xi_{0} \in \mathbf{R}^{n} . \hat{\mu}$ is called very slowly decreasing if there exists a constant $m$ and for each $\varepsilon>0$ a constant $B_{\varepsilon}$ such that

$$
\sup _{\left|\xi-\xi_{0}\right| \leq \varepsilon \log \left(2+\left|\xi_{0}\right|\right)}|\hat{\mu}(\xi)| \geq B_{\varepsilon}\left(1+\left|\xi_{0}\right|\right)^{-m}
$$

for all $\xi_{0} \in \mathbf{R}^{n}$. 
We sketch the proof of this result for the space $\mathcal{E}$ in the Appendix; the given direct proof of the sufficiency of the slowly decreasing condition is due to J. E. Björk (personal communication).

In this note $(\S \S 2-4)$ we prove the following theorems:

THEOREM 1. Let $\mu=\nu_{1}+\nu_{2}$, where $\nu_{1}, \nu_{2} \in \mathcal{E}^{\prime}$ have disjoint singular supports and assume that $\hat{\nu}_{1}$ is slowly decreasing. Then $\hat{\mu}$ is slowly decreasing.

THEOREM 2. Let $\mu \in \mathcal{E}^{\prime}$, let $f$ be real analytic in a neighbourhood of the singular support of $\mu$ and assume $(f \cdot \mu)^{\wedge}$ is slowly decreasing. Then $\hat{\mu}$ is slowly decreasing.

THEOREM 3. Let $\mu \in \mathcal{E}^{\prime}$ be a measure containing an atom (i.e. $\mu\left\{x_{0}\right\} \neq$ 0 for some $x_{0} \in \mathbf{R}^{n}$ ). Then $\hat{\mu}$ is very slowly decreasing.

REMARK 1. I do not know whether Theorems 1 and 2 remain true with "slowly decreasing" replaced by "very slowly decreasing"; the given proofs show they do if $\mu$ is a measure and $\hat{\nu}_{1}$ (resp. $(f \cdot \mu) \hat{)}$ ) is very slowly decreasing in the sense of Definition 1 with $m=0$.

REMARK 2. Measures with non-empty singular support but without an atom may fail to be invertible as the following elementary example shows: Let $n=1$, let $\varphi$ be a test function equal to 1 near $x=0$ and put $\mu=\varphi \cdot \log |\cdot|$; then $\mu$ is invertible if and only if $\varphi \cdot V p(1 / x)$ is, but $(\varphi \cdot V p(1 / x))^{\hat{(}}(\xi)=\int_{-\infty}^{\xi} \hat{\varphi}\left(\xi^{\prime}\right) d \xi^{\prime}$ is not slowly decreasing.

As a corollary to the theorems, we describe in $\$ 5$ a class of invertible (for $\mathcal{E}$ ) distributions which properly contains the distributions with finite supports (see Ehrenpreis [1] and Hörmander [3], Theorem 4.4).

Finally I would like to thank Professor J. E. Björk for the generous advice I was fortunate to profit from during the work on this paper.

2. Proof of Theorem 1. It is no restriction to assume $\mu$ is a measure with total mass not greater than 1 (otherwise regularise $\mu$ by convoluting it with a suitable invertible distribution, see Ehrenpreis [2]).

Since by adding a test function one does not affect the invertibility of $\mu$ we may also assume that "singular support" in the theorem has been replaced by "support".

Let $\varphi$ be a test function such that

(2) $\varphi=1$ on a neighbourhood of $\operatorname{supp} \nu_{1}$ and $\operatorname{supp} \nu_{2} \cap \operatorname{supp} \varphi=\varnothing$. 
By assumption $(\varphi \cdot \mu)^{\wedge}$ is slowly decreasing: for any $\xi_{0} \in \mathbf{R}^{n}$ there exists $\xi_{1} \in \mathbf{R}^{n}$ such that

$$
\left|\xi_{1}-\xi_{0}\right| \leq A \log \left(2+\left|\xi_{0}\right|\right) \text { and } B\left(1+\left|\xi_{0}\right|\right)^{-m} \leq\left|\int \hat{\varphi}(\xi) \hat{\mu}\left(\xi_{1}-\xi\right) d \xi\right|
$$

with some constants $A, B, m$ and we shall assume $B=1$.

For any $R>0$ we may estimate the part of the integral over the ball $|\xi| \leq R$ by

$$
\|\hat{\varphi}\|_{L^{1}} \cdot \sup \left\{|\hat{\mu}(\xi)|:\left|\xi-\xi_{0}\right| \leq R+A \log \left(2+\left|\xi_{0}\right|\right)\right\}
$$

and the remaining part by $\int_{|\xi| \geq R}|\hat{\varphi}(\xi)| d \xi$; we obtain

$$
\text { (3) }\left(1+\left|\xi_{0}\right|\right)^{-m} \leq\|\hat{\varphi}\|_{L^{1}} \cdot \sup _{\left|\xi-\xi_{0}\right| \leq R+A \log \left(2+\left|\xi_{0}\right|\right)}|\hat{\mu}(\xi)|+\int_{|\xi| \geq R}|\hat{\varphi}(\xi)| d \xi \text {. }
$$

We may now pass to infimum over all $\varphi$ satisfying (2). To do this we need

LEMMA 1. Let $\Phi$ be any test function with property (2). Denote by $\mathcal{F}$ the set of all test functions $\varphi$ which satisfy (2) and are such that $\|\hat{\varphi}\|_{L^{1}} \leq\|\hat{\Phi}\|_{L^{1}}$. Then there exist constants $C_{1}, C_{2}>0$ such that, for any $R>0$,

$$
\inf _{\varphi \in \mathscr{F}} \int_{|\xi| \geq R}|\hat{\varphi}(\xi)| d \xi \leq C_{1} e^{-C_{2} R} .
$$

By Lemma 1 with $R=N A \log \left(2+\left|\xi_{0}\right|\right)$, the constant $N$ to be determined shortly, it follows from (3) that

$$
\left(1+\left|\xi_{0}\right|\right)^{-m} \leq\|\hat{\Phi}\|_{L^{1}} \cdot \sup _{\left|\xi-\xi_{0}\right| \leq(N+1) A \log \left(2+\left|\xi_{0}\right|\right)}|\hat{\mu}(\xi)|+C_{1}\left(2+\left|\xi_{0}\right|\right)^{-C_{2} N A}
$$

implying

$$
\sup _{\left|\xi-\xi_{0}\right| \leq\left(m / C_{2}+A\right) \log \left(2+\left|\xi_{0}\right|\right)}|\hat{\mu}(\xi)| \geq B\left(1+\left|\xi_{0}\right|\right)^{-m}
$$

for a suitable constant $B$ if $N$ was chosen so that $C_{2} N A>m$.

Proof of Lemma 1. Suitably chosen positive constants occurring in the proof will all be denoted by $C$. We shall assume with no loss of generality that $R \geq 1$.

Since for all test functions $\varphi$ and all $N=0,1, \ldots$,

$$
\left|\xi_{j}\right|^{N} \cdot|\hat{\varphi}(\xi)| \leq\left\|D_{j}^{N} \varphi\right\|_{L^{1}}, \quad 1 \leq j \leq n,
$$


and since $|\xi|^{N} \leq C^{N} \cdot \Sigma\left|\xi_{j}\right|^{N}, \xi \in \mathbf{R}^{n}$, we easily see that

$$
\int_{|i| \geq R}|\hat{\varphi}| \leq C^{N} R^{n-N} \sum_{j}\left\|D_{j}^{N} \varphi\right\|_{L^{1}}, \quad N>n .
$$

For each such $N$ let $\varphi_{N}$ be a function in $\mathscr{F}$ with the property

$$
\left\|D_{j}^{N} \varphi_{N}\right\|_{L^{1}} \leq C^{N+1} \cdot N !, \quad 1 \leq j \leq n
$$

for example, for a non-negative test function $\psi$ with sufficiently small support and $\int \psi=1$, put $\varphi_{N}=\psi_{(N)} * \cdots * \psi_{(N)} * \Phi$, where $\psi_{(N)}(x)=$ $N^{n} \psi(N x)$ occurs in the convolution $N$ times.

Then

$$
\inf _{\varphi \in \mathscr{F}} \int_{\mid \hat{|s| \geq R}}|\hat{\varphi}| \leq \int_{|\xi| \geq R}\left|\hat{\varphi}_{N}\right| \leq R^{n} \cdot C \cdot\left(\frac{C}{R}\right)^{N} \cdot N !
$$

for $N>n$ and, since $R \geq 1$, also for $N=0,1, \ldots, n$.

Now, for each $N$, take the inverse of (4), multiply it by $2^{-N}$ and then sum over all $N \geq 0$; we obtain

$$
\inf _{\varphi \in \mathscr{F}} \int_{|\xi| \geq R}|\hat{\varphi}| \leq R^{n} \cdot C \cdot e^{-C R}
$$

which is clearly bounded by $C_{1} e^{-C_{2} R}$ for some constants $C_{1}, C_{2}>0$.

3. Proof of Theorem 2. The proof of Theorem 1 applies almost verbatim with condition (2) replaced by

$(2)^{\prime} \quad \varphi=1$ on a neighbourhood of supp $\mu$ and $f$ real analytic on supp $\varphi$, and then $\varphi$ and $\varphi_{N}$ in Lemma 1 replaced by $f \cdot \varphi$ and $f \cdot \varphi_{N}$, respectively.

4. Proof of Theorem 3. We may clearly assume $x_{0}=0$.

Let $\varphi$ be a non-negative test function with support contained in the unit ball in $\mathbf{R}_{\xi}^{n}$ and $\int \varphi=1$.

For $R>0$ put $\varphi_{R}(\xi)=R^{-n} \varphi\left(R^{-1} \xi\right)$; observe that the equalities $\hat{\varphi}_{R}(x)$ $=\hat{\varphi}(R x)$ and $\hat{\varphi}(0)=1$ imply that the functions $\hat{\varphi}_{R}$ converge pointwise to $\chi_{\{0\}}(=$ the characteristic function of the set $\{0\})$ as $R \rightarrow \infty$.

By a direct calculus we see that

$$
\lim _{R \rightarrow \infty} \int \varphi_{R}\left(\xi^{\prime}\right) \hat{\mu}\left(\xi-\xi^{\prime}\right) d \xi^{\prime}=\mu\{0\}
$$


uniformly in $\xi \in \mathbf{R}^{n}$ :

$$
\begin{aligned}
\varphi_{R} * \hat{\mu}(\xi)-\mu\{0\} & =\hat{\varphi}_{R} \cdot \mu\left(e^{-\langle\cdot, \xi\rangle}\right)-\mu\left(\chi_{\{0\}}\right) \\
& =\mu\left(\hat{\varphi}_{R} \cdot e^{-i\langle\cdot, \xi\rangle}-\chi_{\{0\}} \cdot e^{-i\langle\cdot, \xi\rangle}\right),
\end{aligned}
$$

and this is bounded by

$$
\int\left|\hat{\varphi}_{R}-\chi_{\{0\}}\right| d|\mu|
$$

which is clearly convergent to zero as $R \rightarrow \infty$.

It now follows from (5) that, for some $R>0$,

$$
\sup _{\left|\xi-\xi_{0}\right| \leq R}|\hat{\mu}(\xi)| \geq\left|\int \varphi_{R}(\xi) \hat{\mu}\left(\xi_{0}-\xi\right) d \xi\right| \geq \frac{1}{2}|\mu\{0\}|
$$

for all $\xi_{0} \in \mathbf{R}^{n}$.

\section{A class of invertible distributions.}

THEOREM 4. Let $\mu \in \mathcal{E}^{\prime}$ be a measure with an atom, let $\nu \in \mathcal{E}^{\prime}$ have singular support disjoint from that of $\mu$ and let $P$ be a non-zero polynomial. Then $P \cdot \hat{\mu}+\hat{\nu}$ is slowly decreasing.

Proof. By Theorems 1 and 3 all we need to prove is that non-zero polynomials are (very) slowly decreasing: for any $\varepsilon>0$ the function

$$
\mathbf{R}^{n} \ni \xi_{0} \mapsto \int_{|\xi| \leq \varepsilon}\left|P\left(\xi_{0}+\xi\right)\right|^{2} d \xi
$$

is a polynomial with no real zeroes, hence it is bounded away from zero. Therefore, for some $B_{\varepsilon}, C_{\varepsilon}>0$,

$$
\sup _{\left|\xi-\xi_{0}\right| \leq \varepsilon}|P(\xi)| \geq C_{\varepsilon} \cdot\left(\int_{|\xi| \leq \varepsilon}\left|P\left(\xi_{0}+\xi\right)\right|^{2} d \xi\right)^{1 / 2} \geq B_{\varepsilon} .
$$

Appendix. We briefly sketch the proof of the following result of Ehrenpreis [2].

The mapping

$$
\mathcal{E} \exists u \mapsto \check{\mu} * u \in \mathcal{E}
$$

is surjective if and only if $\hat{\mu}$ is slowly decreasing.

Since the adjoint of (A1),

$$
\mathcal{E}^{\prime} \ni \nu \mapsto \mu * \nu \in \mathcal{E}^{\prime},
$$


is injective, $\check{\mu} * \mathscr{E}$ is dense in $\mathscr{E}$; it is equal to $\mathscr{E}$ if and only if $\mu * \mathcal{E}^{\prime}$ is weak* closed (see, for example, Kelley and Namioka [4], Theorem 2.19). By reflexivity of $\mathcal{E}$ the weak* closure of $\mu * \mathcal{E}^{\prime}$ is equal to its weak closure and therefore also to its strong closure, the strong topology of $\mathcal{E}^{\prime}$ being locally convex. Malgrange [5], Corollary on p. 310, proved that $\mu * \mathcal{E}^{\prime}$ is strongly closed if and only if $\hat{\mu}$ has the following division property:

(A3) if $\nu \in \mathcal{E}^{\prime}$ and $\hat{\nu} / \hat{\mu}$ is entire, then $\nu=\mu * \gamma$ for some $\gamma \in \mathcal{E}^{\prime}$.

We now show that (A3) holds if and only if $\hat{\mu}$ is slowly decreasing.

If $\hat{\mu}$ is slowly decreasing then, without losing generality, we may assume that for every $\xi_{0} \in \mathbf{R}^{n}$ there exists $\xi_{1} \in \mathbf{R}^{n}$ such that

$$
\left|\xi_{1}-\xi_{0}\right| \leq A \log \left(2+\left|\xi_{0}\right|\right) \text { and }\left|\hat{\mu}\left(\xi_{1}\right)\right| \geq 1 \text {. }
$$

Let $\nu \in \mathcal{E}^{\prime}$ and assume $\hat{\nu} / \hat{\mu}$ is entire. For $\tau \in \mathbf{C}$ put $\varphi(\tau)=$ $\hat{\mu}\left(\xi_{1}+2 \tau\left(\xi_{0}-\xi_{1}\right)\right)$ and $\psi(\tau)=\hat{\nu}\left(\xi_{1}+2 \tau\left(\xi_{0}-\xi_{1}\right)\right)$. By Harnack's inequality

$$
\log ^{+}\left|\frac{\hat{v}}{\hat{\mu}}\left(\xi_{0}\right)\right|=\log ^{+}\left|\frac{\psi}{\varphi}\left(\frac{1}{2}\right)\right| \leq 3 \cdot \int_{|\tau|=1} \log ^{+}\left|\frac{\psi}{\varphi}\right| .
$$

By subadditivity of $\log ^{+}$and the equality $\log |\varphi|=\log ^{+}|\varphi|-\log ^{+}|1 / \varphi|$, we may estimate the integral in (A4) first by

$$
\int_{|\tau|=1}\left(\log ^{+}|\psi|+\log ^{+}|\varphi|\right)-\int_{|\tau|=1} \log |\varphi|,
$$

and then only by

$$
\int_{|\tau|=1}\left(\log ^{+}|\psi|+\log ^{+}|\varphi|\right)
$$

because, by the assumption,

$$
\int_{|\tau|=1} \log |\varphi| \geq \log |\varphi(0)|=\log \left|\hat{\mu}\left(\xi_{1}\right)\right| \geq 0 .
$$

Since the points on the circle $|\tau|=1$ lie at a distance at most $2 A \log \left(2+\left|\xi_{0}\right|\right)$ from the real space $\mathbf{R}_{\xi}^{n}$ and we have an estimate on $\hat{\mu}$ and $\hat{\nu}$ in terms of the exponential of that distance, the integral (A5) is not greater than $\log C+N \log \left(1+\left|\xi_{0}\right|\right)$ for some constants $C, N$. Thus

$$
\left|\frac{\hat{\nu}}{\hat{\mu}}\left(\xi_{0}\right)\right| \leq C\left(1+\left|\xi_{0}\right|\right)^{N}, \quad \xi_{0} \in \mathbf{R}^{n},
$$

proving that $\hat{\nu} / \hat{\mu}$ has polynomially bounded growth on $\mathbf{R}_{\xi}^{n}$ and therefore, being necessarily of exponential type (see Malgrange [5]), is a FourierLaplace transform of some $\gamma \in \mathcal{G}^{\prime}$. 
Conversely, if $\hat{\mu}$ is not slowly decreasing, then there exists a sequence $\xi_{j} \in \mathbf{R}^{n}, j=1,2, \ldots$, such that

$$
|\hat{\mu}(\xi)|<\left|\xi_{j}\right|^{-j} \quad \text { when }\left|\xi-\xi_{j}\right| \leq j \log \left|\xi_{j}\right|
$$

and we may assume $\left|\xi_{j}\right| \rightarrow \infty$ suitably quickly. It is now possible to construct an entire function $g$ which itself is not a Fourier-Laplace transform of any $\gamma \in \mathcal{E}^{\prime}$, but becomes one when multiplied by $\hat{\mu}$. We indicate the idea: for each $j$ we let $\varphi_{j}$ be a test function with support in a fixed set $k$ such that $\hat{\varphi}_{j}(\xi)$ is about the size of $\left|\xi_{,}\right\rangle$when $\xi=\xi_{J}$, but is conveniently small when $\left|\xi-\xi_{j}\right| \geq j \log \left|\xi_{j}\right|$. The function $g=\sum \hat{\varphi}_{l}$ is of exponential type but not polynomially bounded on $\mathbf{R}_{\xi}^{n}$. At the same time $\hat{\mu} \cdot g=\sum \hat{\mu} \hat{\rho}_{j}$ is polynomially bounded on $\mathbf{R}_{\xi}^{n}$ because $\hat{\mu}$ is small where $\hat{\varphi}_{j}$ is big. For the details of the construction we refer to Ehrenpreis [2] and Hörmander [3].

Added in proof. I wish to thank Olaf von Grudzinski for bringing my attention to the papers [7], [8] of L. Hörmander and in particular to the fact that Theorem 2 of this note (hence also Theorem 1) is a consequence of Theorem 3 in [8] and Lemma 5.4 in [7]. It may be remarked, however, that the proof presented here is independent of the much more advanced methods of [7].

\section{REFERENCES}

[1] L. Ehrenpreis, Solutions of some problems of division III, Amer. J. Math., 78 (1956), 685-715.

[2] _ Solutions of some problems of division IV, Amer. J. Math., 82 (1960), 522-588.

[3] L. Hörmander, On the range of convolution operators, Ann. Math., 76 (1962), 148-170.

[4] J. K. Kelley and J. Namioka, Linear Topological Spaces, Van Nostrand, Princeton, 1963.

[5] B. Malgrange, Existence et approximation des solutions des équations aux dérivées partielles et des équations de convolution, Ann. Inst. Fourier (Grenoble) 6 (1955-56), 271-355.

[6] L. Schwartz, Théroie des distributions, I-II, Paris, 1950-51.

[7] L. Hörmander, Supports and singular supports of convolutions, Acta Math., 110 (1963), 279-302.

[8] __ A remark on singular supports of convolutions, Math. Scand., 45 (1979), 50-54.

Received April 7, 1982.

UNIVERSITY OF STOCKHOLM

Box 6701

S-133 85 StockHOLM

SWEDEN 



\section{PACIFIC JOURNAL OF MATHEMATICS \\ EDITORS}

DONALD BABBITT (Managing Editor)

University of California

Los Angeles, CA 90024

Hugo RossI

University of Utah

Salt Lake City, UT 84112

C. C. MOOre and ARThur OGus

University of California

Berkeley, CA 94720
J. Dugundi

Department of Mathematics

University of Southern California

Los Angeles, CA 90089-1113

R. FINN and H. SAMELSON

Stanford University

Stanford, CA 94305

ASSOCIATE EDITORS

$\begin{array}{lllll}\text { R. Arens } & \text { E. F. Beckenbach } & \text { B. H. Neumann } & \text { F. Wolf } & \text { K. Yoshida }\end{array}$ $(1906-1982)$

\section{SUPPORTING INSTITUTIONS}

UNIVERSITY OF ARIZONA

UNIVERSITY OF BRITISH COLUMBIA

CALIFORNIA INSTITUTE OF TECHNOLOGY

UNIVERSITY OF CALIFORNIA

MONTANA STATE UNIVERSITY

UNIVERSITY OF NEVADA, RENO

NEW MEXICO STATE UNIVERSITY

OREGON STATE UNIVERSITY
UNIVERSITY OF OREGON

UNIVERSITY OF SOUTHERN CALIFORNIA

STANFORD UNIVERSITY

UNIVERSITY OF HAWAII

UNIVERSITY OF TOKYO

UNIVERSITY OF UTAH

WASHINGTON STATE UNIVERSITY

UNIVERSITY OF WASHINGTON 


\section{Pacific Journal of Mathematics}

\section{Vol. 110, No. $1 \quad$ September, 1984}

Wojciech Abramczuk, A class of surjective convolution operators $\ldots \ldots \ldots \ldots 1$

K. Adachi, Extending bounded holomorphic functions from certain

subvarieties of a weakly pseudoconvex domain $\ldots \ldots \ldots \ldots \ldots \ldots$

Malvina Florica Baica, An algorithm in a complex field and its application

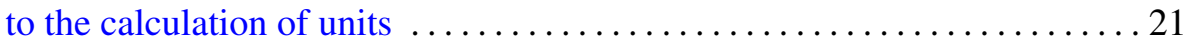

Giuliana Bianchi and Robert Cori, Colorings of hypermaps and a

conjecture of Brenner and Lyndon $\ldots \ldots \ldots \ldots \ldots \ldots \ldots \ldots \ldots \ldots \ldots \ldots$

Ronald James Evans, Determinations of Jacobsthal sums . . .......... 49

Leslie Foged, Characterizations of $\aleph$-spaces .................... 59

Nassif A. Ghoussoub and Paulette Saab, Weak compactness in spaces of

Bochner integrable functions and the Radon-Nikodým property . . . . . . 65

J. Gómez Gil, On local convexity of bounded weak topologies on Banach

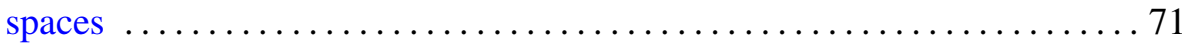

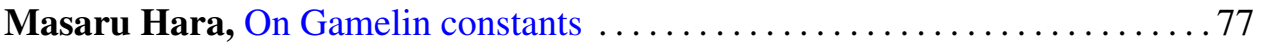

Wilfried Hauenschild, Eberhard Kaniuth and Ajay Kumar, Harmonic

analysis on central hypergroups and induced representations $\ldots \ldots \ldots 83$

Eugenio Hernandez, An interpolation theorem for analytic families of

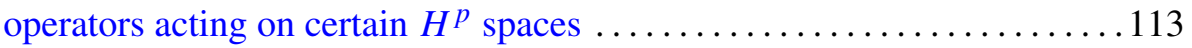

Thomas Alan Keagy, On "Tauberian theorems via block-dominated

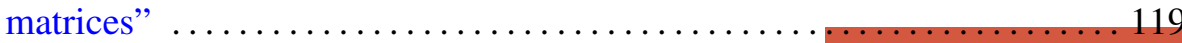

Thomas Landes, Permanence properties of normal structure $\ldots \ldots \ldots \ldots \ldots 125$

Daniel Henry Luecking, Closed ranged restriction operators on weighted

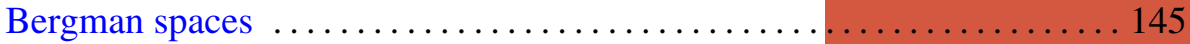

Albert Thomas Lundell, The $p$-equivalence of $\mathrm{SO}(2 n+1)$ and $\mathrm{Sp}(n) \ldots \ldots 161$

Mark D. Meyerson, Remarks on Fenn's "the table theorem" and Zaks' "the chair theorem" ..................................... 167

Marvin Victor Mielke, Homotopically trivial toposes . . . . . . . . . . 171

Gerard J. Murphy, Hyperinvariant subspaces and the topology on Lat A . . 183

Subhashis Nag, On the holomorphy of maps from a complex to a real manifold

Edgar Milan Palmer and Robert William Robinson, Enumeration of self-dual configurations ................................. 203

John J. Walsh and David Clifford Wilson, Continuous decompositions

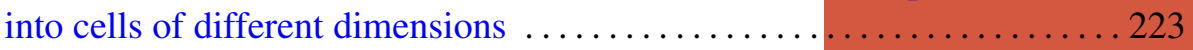

Walter John Whiteley, Infinitesimal motions of a bipartite framework .....233 